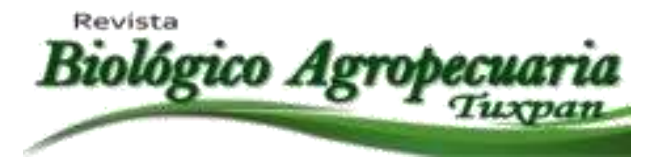

\title{
Caracterización morfometrica de 10 genotipos de nopales productores de Xoconostles (Opuntia spp.) de Hidalgo y Zacatecas México
}

Morphometric characterization of 10 genotypes of producers xoconostle nopales (Opuntia sp.) in Hidalgo and Zacatecas México

Pinedo-Espinoza José Manuel ${ }^{1}$, Clemente Gallegos-Vázquez ${ }^{2}$, Angélica Trapala-Islas ${ }^{3}$, Ambrosio Franco-Bañuelos ${ }^{1}$ y Alma Delia Hernández-Fuentes ${ }^{3 凶}$

${ }^{1}$ Unidad Académica de Agronomía de la Universidad Autónoma de Zacatecas, km 15.5 Carretera Zacatecas-Guadalajara, C.P. 98170, Zacatecas, México. ${ }^{2}$ Centro Regional Universitario Centro Norte de la Universidad Autónoma Chapingo. Calle del Sur Núm. 100 Col. Constelación, poblado El Orito, Zacatecas. CP 98085. ${ }^{3}$ Centro de Investigación en Ciencia y Tecnología de Alimentos, Instituto de Ciencias Agropecuarias, UAEH. km 1 Av. Universidad, Rancho Universitario C.P. 43000, Tulancingo de Bravo Hidalgo, México.

${ }^{\square}$ Autor para correspondencia: $\underline{\text { hfad@hotmail.com }}$

Recibido: $17 / 08 / 2013$

Aceptado: 03/12/2013

\section{RESUMEN}

Se evaluaron 10 genotipos de xoconostle de los estados de Hidalgo y Zacatecas, con el objetivo de realizar una caracterización para potenciar su cultivo, uso y comercialización. La mayoría de los frutos de xoconostle evaluados presentaron un color irregular en cáscara o piel, densidad de areolas media, número de glóquidas de baja a media y de color amarillo, la mayor relación longitud-diámetro se observó en xoconostle 'Borrego', menor longitud del pedúnculo y mayor longitud ecuatorial (diámetro del fruto) en xoconostles 'Cambray' y 'Manzano', pocas semillas en 'Manso' y 'Guinda', mayor porcentaje de mesocarpio (pulpa) y menor porcentaje de pericarpio (cáscara) en 'Borrego' y los frutos de Xoconostle 'Virgen' y 'Cambray' presentaron el menor porcentaje de endocarpio. Al realizar la cosecha de los frutos, tomando como índice de cosecha la menor depresión de la cicatriz del receptáculo, los frutos alcanzaron su madurez óptima de consumo. En años recientes, se ha incrementado la industrialización de productos a base de xoconostle, por lo cual es importante su conservación y la continuación de estudios que ayuden a entender mejor los beneficios que pueden aportar los diversos componentes del xoconostle en la dieta humana.

Palabras clave: características físicas, genotipo, xoconostle.

\section{Revista Científica Biológico Agropecuaria Tuxpan 1 (2) ISSN: 2007-6940}




\begin{abstract}
10 genotypes xoconostle states of Hidalgo and Zacatecas, in order to perform a characterization to enhance cultivation, use and trade were evaluated. Most fruits xoconostle evaluated showed an irregular peel or skin color, density medium areolas, glochids number of low to medium and yellow, the greater length to diameter ratio was observed in xoconostle ' Borrego ' shorter peduncle and longer equatorial ( fruit diameter ) in xoconostles ' Cambray ' and' Manzano ', few seeds in ' Meek ' and' Cherry ', a higher percentage of mesocarp (pulp) and lower percentage of pericarp ( husk) at' Borrego ' and the fruits of Xoconostle ' Virgin ' and' Cambray 'showed the lowest percentage of endocarp. When the fruit harvest, harvest index taking as minor depression scar receptacle, the fruits reached their optimum ripening. In recent years, increased industrialization xoconostle based products, which is important for its preservation and continuation of studies to help better understand the potential benefits xoconostle the various components of the human diet.
\end{abstract}

Key words: physical characteristics, genotype, xoconostle.

\section{INTRODUCCIÓN}

El fruto de xoconostle presenta una cáscara muy gruesa de $1.5 \mathrm{~cm}$ de espesor, es ácido (agridulce) y con poca pulpa (Colunga et al., 1986; Reyes-Agüero et al., 2006). A la fecha se han identificado ocho especies de xoconostle que crecen de forma silvestre, en regiones semiáridas de México, pero otras especies son cultivadas en jardines y plantaciones comerciales (Vigueras y Portillo, 2001; Casas y Barbera, 2002; Reyes Agüero et al., 2005). En México se cultivan 1,031 hectáreas de xoconostle con una producción de 10,148 toneladas, de las cuales el estado de Hidalgo produce 184 toneladas, lo que representa $1.87 \%$ de xoconostle total (SAGARPA, 2005).

En México se cultivan 1,031 hectáreas de xoconostle y su producción es de 10,148 toneladas totales. En Pachuca, su producción es de 184 toneladas, lo que representa $1.87 \%$ de xoconostle total (SAGARPA, 2005). En años recientes, se ha incrementado la industrialización de productos a base de xoconostle y han llamado la atención de los mercados internacionales, comenzando a mejorarse los precios en nuestro país. Con base en lo anterior en el presente trabajo se planteó realizar la caracterización de variedades y selecciones de Xoconostle para potenciar su cultivo, uso y comercialización.

\section{MATERIALES Y MÉTODOS}

Los frutos de las variedades 'Cuerón', 'Cambray', 'Virgen' y selección 'Cuaresmeño Zacatecano' fueron cosechados en el estado de Zacatecas, mientras que los de frutos de selecciones 'Matizado', 'Manso', 'Invierno', 'Borrego' y 'Ulapa' en el estado de Hidalgo y 'Manzano' en el estado de México.

\section{Variables de estudio}

Las determinaciones físicas de fruto como longitud, diámetro máximo, relación longitud/diámetro máximo, longitud del pedúnculo, depresión de la cicatriz del

\title{
Revista Científica Biológico Agropecuaria Tuxpan 1 (2)
}


receptáculo y diámetro de la cicatriz del receptáculo, se midieron con un vernier digital marca Mitutoyo. Forma de fruto, densidad de areolas, número y color de glóquidas, longitud del pedúnculo, depresión y diámetro de la cicatriz del receptáculo, uniformidad del color de la superficie, semillas completas y abortivas, se determinaron con base en el descriptor para variedades de tuna propuesto por Gallegos et al. (2005). El color de cáscara se evaluó con un colorímetro "Hunter L, a, b". Para la determinación de peso de pulpa, cascara y semillas del fruto, este se cortó y separo de acuerdo a: pulpa (mesocarpio), cáscara (exocarpio) y semillas (endocarpio), colocando cada una de las partes sobre una báscula digital marca OHAUS.

\section{RESULTADOS Y DISCUSIÓN}

Los frutos de xoconostles 'Manso', 'Cuaresmeño Zacatecano' y 'Cueron' presentaron un color uniforme en la cáscara (Cuadro 1). La mayoría de los consumidores prefieren frutos con color uniforme y es el primer criterio que toman en cuenta para seleccionarlos.

Cuadro 1. Uniformidad de color de la cáscara de frutos de xoconostle, número de semillas desarrolladas y número de semillas abortivas.

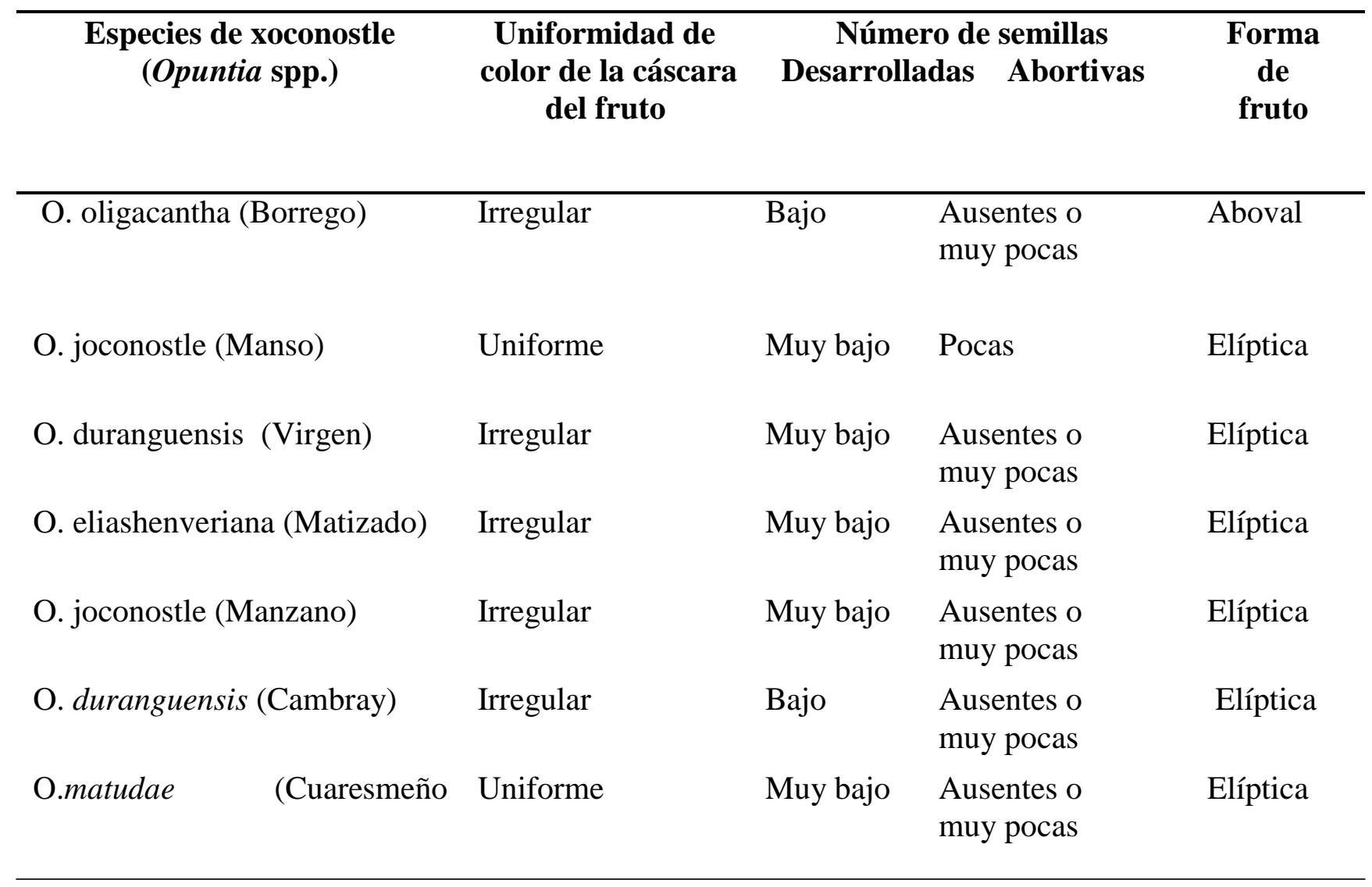


Zacatecano)

\begin{tabular}{|c|c|c|c|c|}
\hline O. matudae (Cuerón) & Uniforme & Muy bajo & $\begin{array}{l}\text { Ausentes o } \\
\text { muy pocas }\end{array}$ & Elíptica \\
\hline O. tezontepecana (Invierno) & Irregular & Muy bajo & Pocas & Elíptica \\
\hline
\end{tabular}

O.joconostle (Ulapa) $\quad$ Irregular $\quad$ Medio $\quad$ Pocas $\quad$ Aboval

La mayoría de los frutos presentaron un número se semillas desarrolladas muy bajo y semillas abortivas ausentes ó muy pocas, excepto los frutos de xoconostle 'Borrego' y 'Cambray' que presentaron un numero de semillas desarrolladas bajo, y los frutos de xoconostle 'Invierno' y 'Ulapa' presentaron pocas semillas abortivas y número de semillas desarrolladas muy bajo y medio. La forma de los frutos fue elíptica excepto para los frutos de xoconostle 'Borrego' y 'Ulapa' que presentaron una forma aboval. García-Pedroza, et al.
(2005), observaron una forma globosa y ovoide en seis cultivares y variedades de xoconostle.

La densidad de areolas fue baja, media y alta y el número de glóquidas baja y media en los xoconostles evaluados. En los frutos de xoconostle 'Borrego, 'Manso', 'Invierno' y 'Ulapa', la densidad de areolas fue baja y media y el número de glóquidas bajo, lo cual permite que el desespinado sea más fácil (Cuadro 2).

Cuadro 2. Características de densidad de areolas, número de glóquidas y color de glóquidas de frutos de xoconostle (Opuntia spp.).

\begin{tabular}{llll}
\hline $\begin{array}{c}\text { Especies de xoconostle } \\
\text { (Opuntia spp.) }\end{array}$ & $\begin{array}{c}\text { Densidad } \\
\text { de areolas }\end{array}$ & $\begin{array}{c}\text { Número de } \\
\text { glóquidas }\end{array}$ & $\begin{array}{c}\text { Color de } \\
\text { glóquidas }\end{array}$ \\
\hline Borrego & Baja & Baja & Amarillas \\
Manso & Baja & Baja & Amarillas \\
Virgen & Media & Media & Amarillas \\
Matizado & Media & Media & Marrón \\
\hline
\end{tabular}

Revista Científica Biológico Agropecuaria Tuxpan 1 (2) 


$\begin{array}{llcl}\text { Manzano } & \text { Media } & \text { Baja } & \text { Amarillo } \\ \text { Cambray) } & \text { Alta } & \text { Baja } & \text { Amarillas } \\ \text { Cuaresmeño Zacatecano } & \text { Alta } & \text { Baja } & \text { Amarillas } \\ \text { Cuerón } & \text { Media } & \text { Media } & \text { Amarillas } \\ \text { Invierno } & \text { Media } & \text { Baja } & \text { Marrón } \\ \text { Ulapa } & \text { Media } & \text { Baja } & \text { Amarillas }\end{array}$

Con respecto a las características morfoagronómicas, se observaron diferencias estadísticas significativas en la relación longitud/diámetro, longitud del pedúnculo, depresión de la cicatriz estilar y diámetro de la cicatriz del receptáculo (Cuadro 3). Los xoconostles 'Borrego' y 'Ulapa' presentaron una mayor relación longitud/diámetro y no se observaron diferencias estadísticas significativas entre ellos $(1.58$ y $1.69 \mathrm{~cm})$ y fueron los frutos de mayor tamaño, sin embargo los frutos de xoconostle 'Cambray' y 'Ulapa' presentaron mayor diámetro de cicatriz del receptáculo. La composición de los frutos varia con la madurez y hay que tener en cuenta que son frutos "no climatéricos", por lo que es importante cosecharlos en el punto de madurez óptimo de consumo; se han propuesto diferentes parámetros por definir la mejor época de cosecha del fruto; tamaño, y llenado del receptáculo del fruto, cambio de color de la cáscara y profundidad de la cavidad del receptáculo entre otros. La mayoría de los productores utilizan como índice de cosecha la depresión de la cicatriz del receptáculo, por eso es importante conocer las variaciones que existen en los diferentes frutos de xoconostles evaluados.

Cuadro 3. Características morfoagronómicas de frutos de xoconostle (Opuntia spp.)

\begin{tabular}{|c|c|c|c|c|}
\hline $\begin{array}{l}\text { Especies de xoconostle } \\
\text { (Opuntia spp.) }\end{array}$ & $\begin{array}{l}\text { Relación } \\
\text { longitud/ } \\
\text { diámetro } \\
\text { (cm) }\end{array}$ & $\begin{array}{l}\text { Longitud } \\
\text { del } \\
\text { pedúnculo } \\
\text { (cm) }\end{array}$ & $\begin{array}{c}\text { Depresión } \\
\text { de } \\
\text { la cicatriz } \\
\text { estilar } \\
\text { (cm) }\end{array}$ & $\begin{array}{l}\text { Diámetro } \\
\text { de } \\
\text { la cicatriz } \\
\text { del } \\
\text { receptáculo } \\
\text { (cm) }\end{array}$ \\
\hline Borrego & $1.58 \mathrm{a}^{\mathrm{Z}}$ & $0.031 \mathrm{~cd}$ & $0.047 \mathrm{cde}$ & $0.263 b c$ \\
\hline
\end{tabular}




$\begin{array}{lcccc}\text { Manso } & 1.33 \mathrm{bc} & 0.032 \mathrm{~cd} & 0.060 \mathrm{bc} & 0.212 \mathrm{e} \\ \text { Virgen } & 1.00 \mathrm{~d} & 0.029 \mathrm{~d} & 0.056 \mathrm{~cd} & 0.276 \mathrm{abc} \\ \text { Matizado } & 1.29 \mathrm{bc} & 0.104 \mathrm{a} & 0.087 \mathrm{a} & 0.224 \mathrm{e} \\ \text { Manzano } & 1.23 \mathrm{c} & 0.028 \mathrm{~d} & 0.071 \mathrm{~b} & 0.230 \mathrm{de} \\ \text { Cambray } & 1.09 \mathrm{~d} & 0.053 \mathrm{~b} & 0.044 \mathrm{e} & 0.290 \mathrm{ab} \\ \text { Cuaresmeño Zacatecano } & 1.25 \mathrm{bc} & 0.044 \mathrm{bc} & 0.054 \mathrm{~cd} & 0.256 \mathrm{~cd} \\ \text { Cuerón } & 1.24 \mathrm{c} & 0.039 \mathrm{~cd} & 0.051 \mathrm{cde} & 0.269 \mathrm{abcd} \\ \text { Invierno } & 1.38 \mathrm{~b} & 0.039 \mathrm{~cd} & 0.053 \mathrm{~cd} & 0.273 \mathrm{abc} \\ \text { Ulapa } & 1.69^{\mathrm{a}} & 0.037 \mathrm{~cd} & 0.037 \mathrm{e} & 0.298^{\mathrm{a}} \\ \text { DMS } & 0.134 & 1.34 & 1.47 & 2.94 \\ \text { CV (\%) } & 9.43 & 27.94 & 23.78 & 10.56\end{array}$

$\mathrm{Z}_{\text {Valores con las mismas letras dentro de la columna son iguales de acuerdo a la prueba de Tukey a }}$ una $\mathrm{P} \leq 0.05$.

DMS: Diferencia mínima significativa; CV: Coeficiente de variación.

En longitud ecuatorial, longitud polar, porcentaje de cáscara, porcentaje de pulpa y porcentaje de semillas se observaron diferencias significativas (Cuadro 4).

Los frutos de xoconostle 'Invierno', 'Cueron' y 'Cambray' presentaron la mayor longitud ecuatorial $(4.96,4.77$ y $4.71 \mathrm{~cm})$ y los valores fueron mayores a los reportados por García -Pedroza, et al. (2005), mientras que los frutos de xoconostle 'Ulapa', 'Invierno' y
'Borrego' presentaron la mayor longitud polar (7.7, 6.83 y 6.58) y estos valores también fueron superiores a lo reportado por García Pedroza, et al. (2005).

El mayor porcentaje de pulpa (mesocarpio) se observó en los xoconostles 'Borrego', 'Manso', 'Cuaresmeño Zacatecano', 'Cambray' y 'Ulapa', (70.73, 61.48, 60.24 y $57.31 \%$ ), estos porcentajes fueron superiores a los reportados por García-Pedroza, et al.

\section{Revista Científica Biológico Agropecuaria Tuxpan 1 (2)}


(2005), ellos reportaron porcentajes de 24.9 a $9.3 \%$. Los frutos con mayor porcentaje de pulpa permiten obtener un mayor rendimiento en la industrialización de productos elaborados con xoconostles (salsas, dulces entre otros). Los xoconostles 'Manzano', 'Virgen' e 'Invierno presentaron el mayor porcentaje de cáscara $(65.19,32.46$ y 31.02$)$ y estos porcentajes fueron menores a los reportados por García Pedroza, et al. (2005). El agua es el componente principal de la fruta y por ello uno de los mayores atractivos para las zonas áridas y semiáridas, el agua se encuentra protegida por la gruesa cáscara, rica en mucilago que retiene fuertemente y contribuyen a la baja deshidratación del fruto.

Cuadro 4. Características morfológicas de frutos de Xoconostle (Opuntia spp.)

\begin{tabular}{|c|c|c|c|c|c|}
\hline $\begin{array}{c}\text { Especies de } \\
\text { xoconostle } \\
\text { (Opuntia spp.) }\end{array}$ & $\begin{array}{c}\text { Longitud } \\
\text { ecuatorial } \\
\text { (Diámetro) } \\
(\mathrm{cm})\end{array}$ & $\begin{array}{l}\text { Longitud } \\
\text { polar } \\
\text { (Largo) } \\
\text { (cm) }\end{array}$ & $\begin{array}{c}\text { Porcentaje } \\
\text { de } \\
\text { cáscara } \\
\text { (pericarpio) } \\
(\%)\end{array}$ & $\begin{array}{c}\text { Porcentaje } \\
\text { de } \\
\text { pulpa } \\
\text { (mesocarpio) } \\
(\%)\end{array}$ & $\begin{array}{c}\text { Porcentaje } \\
\text { de } \\
\text { semillas } \\
\text { (endocarpio) } \\
(\%)\end{array}$ \\
\hline Borrego & $4.23 \mathrm{~d}^{\mathrm{Z}}$ & $6.58 b$ & $12.10 \mathrm{f}$ & $70.73 a$ & $17.15 b c$ \\
\hline Manso & $4.38 \mathrm{~cd}$ & $5.84 \mathrm{c}$ & $26.53 \mathrm{cde}$ & 63.81de & $19.65 b$ \\
\hline Virgen & $4.25 \mathrm{~d}$ & $4.28 \mathrm{e}$ & $32.46 b$ & 55.97cde & $11.56 \mathrm{~d}$ \\
\hline Matizado & $4.37 \mathrm{~cd}$ & $5.65 \mathrm{~cd}$ & $30.21 b c$ & $43.18 f$ & $26.60 \mathrm{a}$ \\
\hline Manzano & $4.45 \mathrm{bcd}$ & $5.47 \mathrm{~cd}$ & $65.19 \mathrm{a}$ & $20.31 \mathrm{~g}$ & $14.48 \mathrm{~cd}$ \\
\hline Cambray & 4.71abc & $5.15 \mathrm{~d}$ & $27.88 \mathrm{cbd}$ & $60.24 b c$ & $11.87 \mathrm{~d}$ \\
\hline Cuaresmeño & $4.54 \mathrm{bcd}$ & $5.72 \mathrm{~cd}$ & 23.66de & $61.48 b$ & $14.84 \mathrm{bcd}$ \\
\hline \multicolumn{6}{|l|}{ Zacatecano) } \\
\hline Cuerón & $4.77 \mathrm{ab}$ & $5.85 \mathrm{c}$ & $22.59 \mathrm{e}$ & $51.75 \mathrm{e}$ & $25.65 a$ \\
\hline Invierno & $4.96 \mathrm{a}$ & $6.83 b$ & $31.02 \mathrm{bc}$ & $53.50 \mathrm{de}$ & $15.47 \mathrm{bcd}$ \\
\hline
\end{tabular}




$\begin{array}{lccccc}\text { Ulapa } & 4.45 \mathrm{bcd} & 7.78 \mathrm{a} & 28.36 \mathrm{bcd} & 57.31 \mathrm{bcd} & 14.31 \mathrm{~cd} \\ \text { DMS } & 3.63 & 5.77 & 5.20 & 5.29 & 5.15 \\ \mathrm{CV}(\%) & 7.36 & 9.13 & 15.48 & 9.18 & 27.59\end{array}$

$\mathrm{Z}_{\text {Valores con las mismas letras dentro de la columna son iguales de acuerdo a la prueba de Tukey a }}$ una $\mathrm{P} \leq 0.05$.

DMS: Diferencia mínima significativa; CV: Coeficiente de variación.

\section{CONCLUSIONES}

En la colecta realizada en los estados de Hidalgo y Zacatecas se encontraron en las diez variedades frutos de diferente color. La mayoría de los frutos de xoconostle evaluados presentaron un color irregular en cáscara o piel, densidad de areolas media, número de glóquidas de baja a media y de color amarillo. Los xoconostles 'Borrego' y 'Ulapa'

presentaron una mayor relación longitud/diámetro. El mayor porcentaje de pulpa (mesocarpio) se observó en los frutos de xoconostle 'Borrego', 'Manso', 'Cuaresmeño Zacatecano', 'Cambray' y 'Ulapa'. En este trabajo se demostró la diversidad de variedades de xoconoslte silvestre, por lo cual se sugiere su conservación y potenciar su uso en las zonas áridas y semiáridas.

\section{AGRADECIMIENTOS}

Al Servicio Nacional de Inspección y Certificación de Semillas (SNICS), Sistema Nacional de Recursos Fitogenéticos (SINAREFI), SAGARPA y CONACYT por el apoyo para la realización de este trabajo.

\section{LITERATURA CITADA}

Casas, A. and Barbera, G. 2002. Mesoamerican domestication and diffusion. In P. S. Nobel (Ed), Cacti, Biology and uses. Berkeley,Los Angeles. London: University of California press. Pp. 143162.

Colunga G.M.P., Hernández X.E., Castillo A. 1986). Variación morfológica, manejo agrícola y grados de domesticación de Opuntia spp. en el Bajío Guanajuatense Agrociencia 65: 7-49.

Gallegos-Vázquez, C., Cervantes-Herrera J., Barrientos-Priego A. F. 2005. Manual grafico para la descripción varietal del nopal tunero y xoconostle (Opuntia spp). pp. 70-109. ISBN 968-02-0132-5.

Garcia-Pedraza L.G., Reyes Aguero J.A., Aguire-Rivera J.R., y Pinos-Rodriguez J.M. 2005. Preliminary nutritional and organoleptic assessment of xoconostle fruit (Opuntia spp.) as a condiment or appetizer Food. Science 3(17): 333-340.

Reyes-Agüero, J. R.; R. A. Aguirre; A. Valiente-Banuet. 2006. Reproductive biology of Opuntia: A review. Journal of 
Arid Environments 64:549-585.

https://doi.org/10.1016/j.jaridenv.2005.06.018

Secretaría de Agricultura Ganadería Desarrollo

Rural Pesca y Alimentación

(SAGARPA), 2005. Servicio de

información $\quad$ E $\quad$ Estadística

Agroalimentaria y Pesca. Resumen nacional DDR. Año agrícola 2005.

https://doi.org/10.32870/cer.v0i118.7067

Copyright (c) 2013 José Manvel Pinedo Espinoza, Clemente Gallegos Vázquez, Angélica Trapala Is las, Ambrosio Franco Bañuelos y Alma Delia Hemández Fuentes

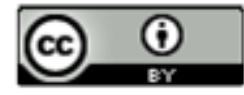

Este texto está protegido por una licencia Creative Commonz 4.0

Usted es libre para Compartir —copiar y redistribuir el material en cualquier medio o formato- y Ad aptar el documento —remezclar, transformar y crear a partir del material- para cualquier propósito, inchso para fines comerciales, siempre que cumpla la condición de:

Atribución: Usted debe dar crédito a la obra original de manera adecuada, proporcionar un enlace a la licencia, e indicar si se han realizad o cambios. Puede hacerio en cualquier forma razonable, pero no de forma tal que sugiera que tiene el apoyo del licenciante o lo recibe por el usoque hace de la obra.

Resumendelicencia - Textocompletodelalicencia

Revista Científica Biológico Agropecuaria Tuxpan 1 (2)

ISSN: 2007-6940 